\title{
Exploring the cardiac response to injury in heart transplant biopsies
}

\author{
Philip F. Halloran, ${ }^{1,2}$ Jeff Reeve, ${ }^{1,3}$ Arezu Z. Aliabadi, ${ }^{4}$ Martin Cadeiras, ${ }^{5}$ Marisa G. Crespo-Leiro, ${ }^{6}$ \\ Mario Deng, ${ }^{5}$ Eugene C. Depasquale, ${ }^{5}$ Johannes Goekler, ${ }^{4}$ Xavier Jouven, ${ }^{7}$ Daniel H. Kim, ${ }^{3}$ \\ Jon Kobashigawa, ${ }^{8}$ Alexandre Loupy, ${ }^{9}$ Peter Macdonald, ${ }^{10}$ Luciano Potena, ${ }^{11}$ Andreas Zuckermann, ${ }^{4}$ \\ and Michael D. Parkes ${ }^{1}$ \\ 'Alberta Transplant Applied Genomics Centre, Edmonton, Alberta, Canada. ${ }^{2}$ Department of Medicine, University of Alberta, \\ Edmonton, Alberta, Canada. ${ }^{3}$ Department of Laboratory Medicine and Pathology, University of Alberta, Edmonton, \\ Alberta, Canada. ${ }^{4}$ Medical University of Vienna, Vienna, Austria. ${ }^{5}$ Ronald Reagan UCLA Medical Center, Los Angeles, \\ California, USA. ${ }^{6}$ Complexo Hospitalario Universitario A Coruña, A Coruña, Spain. ${ }^{7}$ Paris Transplant Group, Paris, France. \\ ${ }^{8}$ Cedars-Sinai Medical Center, Los Angeles, Calfornia, USA. ${ }^{9}$ Hôpital Necker, Paris, France. ${ }^{10}$ The Victor Chang Cardiac \\ Research Institute, Sydney, Australia. "'Cardiovascular Department, University of Bologna, Bologna, Italy.
}

BACKGROUND. Because injury is universal in organ transplantation, heart transplant endomyocardial biopsies present an opportunity to explore response to injury in heart parenchyma. Histology has limited ability to assess injury, potentially confusing it with rejection, whereas molecular changes have potential to distinguish injury from rejection. Building on previous studies of transcripts associated with T cell-mediated rejection (TCMR) and antibody-mediated rejection (ABMR), we explored transcripts reflecting injury.

METHODS. Microarray data from 889 prospectively collected endomyocardial biopsies from 454 transplant recipients at 14 centers were subjected to unsupervised principal component analysis and archetypal analysis to detect variation not explained by rejection. The resulting principal component and archetype scores were then examined for their transcript, transcript set, and pathway associations and compared to the histology diagnoses and left ventricular function.

RESULTS. Rejection was reflected by principal components PC1 and PC2, and by archetype scores $\mathrm{S2}_{\text {TCMR }}$, and $\mathrm{S}_{\mathrm{ABMR}}$, with $\mathrm{S1}_{\text {normal }}$ indicating normalness. $\mathrm{PC}$ and a new archetype score, $\mathrm{S4}_{\text {injury' }}$, identified unexplained variation correlating with expression of transcripts inducible in injury models, many expressed in macrophages and associated with inflammation in pathway analysis. S4 ${ }_{\text {injury }}$ scores were high in recent transplants, reflecting donation-implantation injury, and both S4 ${ }_{\text {injury }}$ and $S 2_{\text {TCMR }}$ were associated with reduced left ventricular ejection fraction.

CONCLUSION. Assessment of injury is necessary for accurate estimates of rejection and for understanding heart transplant phenotypes. Biopsies with molecular injury but no molecular rejection were often misdiagnosed rejection by histology.

TRAIL REGISTRATION. ClinicalTrials.gov NCT02670408

FUNDING. Roche Organ Transplant Research Foundation, the University of Alberta Hospital Foundation, and Alberta Health Services. shares in Transcriptome Sciences Inc., a University of Alberta research company with an interest in molecular diagnostics.

License: Copyright 2018, American Society for Clinical Investigation.

Submitted: July 26, 2018

Accepted: September 11, 2018

Published: October 18, 2018

Reference information: JCI Insight. 2018;3(20):e123674. https://doi.org/10.1172/jci. insight.123674.

\section{Introduction}

Heart transplant endomyocardial biopsies (EMBs) provide an opportunity to characterize pathogenic processes unique to transplants such as rejection but also offer potential insight into states that are of general interest in cardiology such as the parenchymal response to injury. Currently, EMBs are studied by histology to diagnose rejection, following the guidelines of the International Society for Heart and Lung Transplantation (ISHLT) (1-4). The features of $\mathrm{T}$ cell-mediated rejection (TCMR) include interstitial inflammation and myocyte damage, and the features of antibody-mediated rejection (ABMR) include microvascular inflammation, complement factor C4d deposition (5-9), and a positive test for circulating donor-specific anti-HLA antibodies (DSAs) (10-13). However, DSA is present in many patients with no 
ABMR and absent in some patients with ABMR (11-13). In general, histologic features of rejection are more difficult to score reproducibly in EMBs than they are in kidney core biopsies $(14,15)$.

Molecular assessment of transplant biopsies offers the potential for greater precision and accuracy in diagnosing rejection. We recently published a first-generation Molecular Microscope diagnostic system (MMDx-Heart) for histology-independent assessment of EMBs based on their expression of rejection-associated transcripts (RATs) that were originally derived in kidneys (16). Molecular rejection scores were developed using an unsupervised machine learning method called archetypal analysis, which identifies a specified number of extreme phenotypes (archetypes) in the data. The first-generation MMDx-Heart system was based on a 3-archetype analysis (3AA), which gave each biopsy a normalness score $\left(\mathrm{S}_{\text {normal }}\right)$, a TCMR score $\left(S 2_{\text {TCMR }}\right)$, and an ABMR score $\left(S 3_{\mathrm{ABMR}}\right)$ describing its closeness to each of the 3 corresponding archetypes, which correlated with rejection in histology diagnoses, albeit with discrepancies as expected (17). The use of kidney-derived RATs to classify heart transplant biopsies was possible because many transcripts and the corresponding mechanisms in rejection are shared between kidney, heart, and presumably other organ transplants.

In addition to rejection, molecular assessment offers an opportunity to explore the response to injury, and to determine whether injury is being mistaken for rejection in histology. Injured tissue evokes innate immunity (inflammation), and parenchymal injury may contribute to reduced function and ultimately to graft failure. The most recent ISHLT consensus on diagnosis of heart rejection acknowledges that inflammation induced by injury can be confused with rejection, but no standardized criteria exist to score or correct for injury (1). The present study sought to characterize the variance in transcript expression in EMBs that was not explained by rejection (17). We hypothesized that the unexplained variance would be attributable to parenchymal injury and its inflammatory effects (the response to wounding), and that variation in RAT expression could be used to capture injury-induced changes because injury-related innate immunity shares molecular mechanisms with rejection-related adaptive immune responses. Characterization of the unexplained variance involved examining its time course and its relationship to published transcript sets reflecting recent injury in tissues with no rejection: mouse cardiac isografts (18), mouse kidney isografts $(19,20)$, injured human kidney transplants $(21)$, and damage-associated molecular pattern (DAMP) transcripts $(22,23)$. We also studied whether injury was associated with depressed cardiac function and was being confused with rejection by histology. Our study design is outlined in Figure 1.

\section{Results}

Biopsy population and rejection classification. We studied 889 clinically indicated, protocol, and follow-up EMBs (Table 1), including 331 biopsies from the earlier study (17). The 14 contributing centers from 7 countries are listed in Supplemental Table 1 (supplemental material available online with this article; https://doi.org/10.1172/jci.insight.123674DS1). The common primary diseases were cardiomyopathy and coronary artery disease.

Histologic ISHLT diagnoses (Table 2) were expressed as a simplified classification to facilitate comparisons with molecular assessments: TCMR, possible TCMR (pTCMR), ABMR, and possible ABMR (pABMR) (see Methods). Histology classified the EMBs as 334 no rejection (38\%), 51 ABMR (6\%), 63 pABMR (7\%), 84 TCMR (9\%), 273 pTCMR (31\%), 9 mixed ABMR and TCMR (1\%), and 71 mixed pABMR and pTCMR (8\%). Histology diagnoses were unavailable for 4 biopsies. In standard-of-care local HLA antibody testing (Table 2), 158 (37\%) patients were DSA positive at their most recent testing, although DSA at biopsy is not standard-of-care in some centers and were often not assessed.

Unsupervised analyses of unexplained variation in transcript expression. Our goal was to explore molecular changes that were not adequately described by the rejection scores in the 3AA-based first-generation MMDx-Heart diagnostic system (17). We analyzed unexplained variation (UV) in the expanded cohort of 889 EMBs by re-deriving the molecular scores using a 4-archetype model (4AA) based on RAT expression. The fourth archetype in the 4AA model corresponds to UV, and introduces a corresponding molecular score, $\mathrm{S} 4_{\mathrm{UV}}$. Comparison of the scores for the 4AA versus 3AA models (Supplemental Figure 1) showed that incorporation of the $\mathrm{S} 4_{\mathrm{UV}}$ score had almost no effect on the normalness measurements; $\mathrm{S} 1_{\text {normal }}$ was almost perfectly conserved in 4AA versus 3AA (Spearman's correlation coefficient $\rho_{\mathrm{s}}=1.00$ ). In contrast, the rejection estimates ( $\mathrm{S} 2_{\text {TCMR }}$ and $\mathrm{S} 3_{\mathrm{ABMR}}$ ) were lower when the $\mathrm{S} 4_{\mathrm{UV}}$ score was assigned. Nevertheless, the $\mathrm{S} 2_{\mathrm{TCMR}}$ and $\mathrm{S} 3_{\mathrm{ABMR}}$ in the $4 \mathrm{AA}$ model were highly correlated with those in $3 \mathrm{AA}\left(\rho_{\mathrm{s}}=0.92\right.$ and 0.91 , respectively) and as shown below displayed similar molecular associations. 


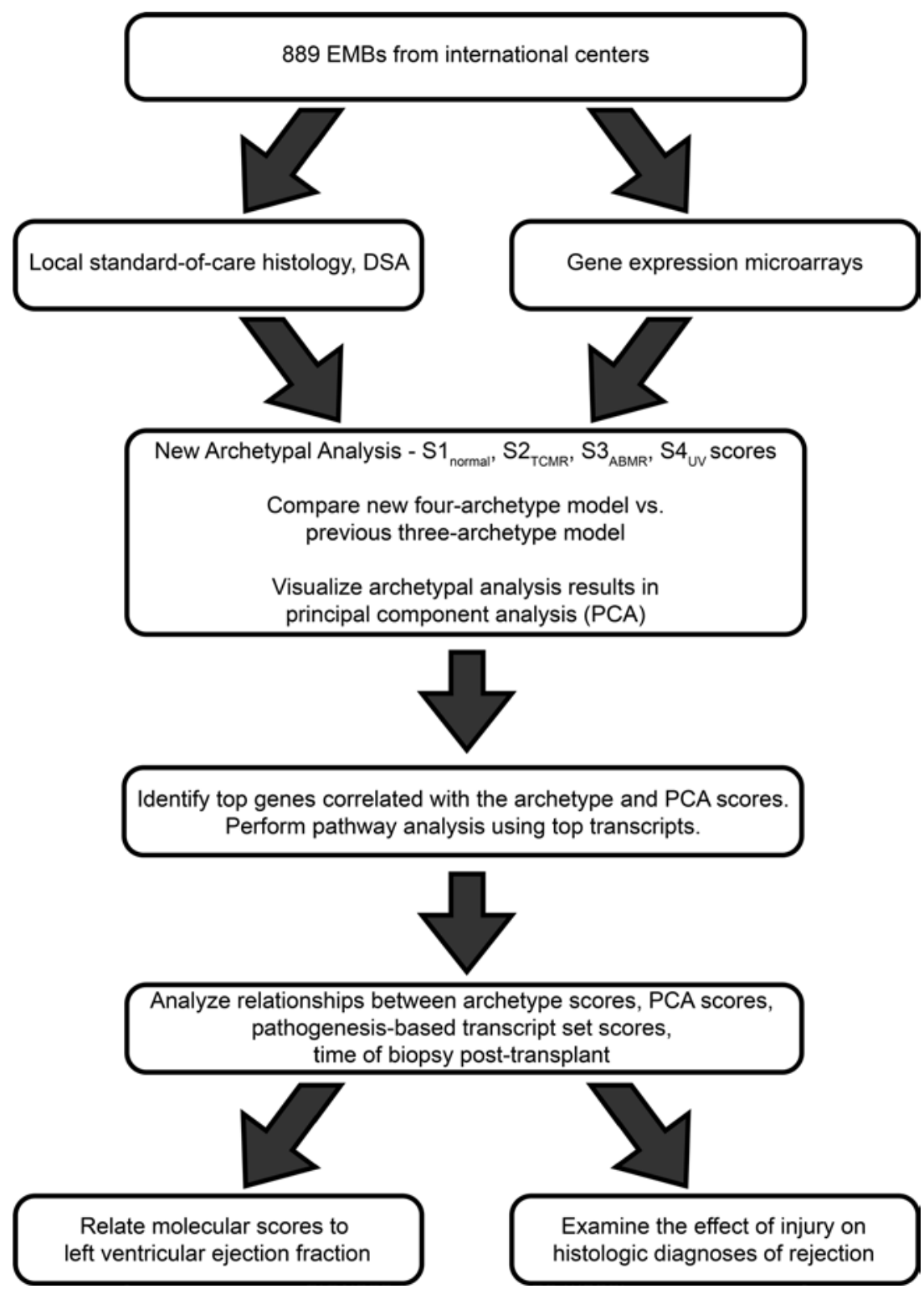

Figure 1. Overview of the work plan implemented in this investigation.

We also explored UV using principal component analysis (PCA) based on RAT expression. In addition to principal components 1 and 2 (PC1 and PC2) studied previously (17), the present study examined the third major source of variation, PC3 (Figure 2). PC1 (62\% of variation) distinguished normal from abnormal biopsies. PC2 (6\% of variation) separated ABMR from TCMR. PC3 (4\% of the variation) distinguished many biopsies from both rejection and normalness. Biopsies with high PC3 scores often had high PC1 scores, but lacked a strong relationship to PC2. PC3 correlated with S4 $4_{\mathrm{UV}}$ scores $\left(\rho_{\mathrm{s}}=0.69\right)$. The data indicate that UV in RAT expression (PC3 and S4 ${ }_{\mathrm{UV}}$ ) represents an abnormal phenotype that is different from ABMR and TCMR.

Top transcripts correlating with the $S 4_{U V}$ score and PC3 score. Whereas the scores from PCA and archetypal analyses were derived solely from expression of RATs in EMBs, the top transcripts correlated with RATbased scores are not necessarily the RATs. We examined the transcripts most strongly correlated with $\mathrm{S} 4_{\mathrm{UV}}$ (Table 3) and PC3 (Supplemental Table 2), and annotated their expression in a panel of cultured human cells. Only 1 of 30 of the top PC3-correlated transcripts and 9 of 30 top S4 ${ }_{\mathrm{UV}}$-correlated transcripts were RATs. In contrast, RATs were prominent in the top 30 transcripts correlating with the rejection-related scores: 20 of 30 for PC1, 12 of 30 for PC2, 22 of 30 for S1 ${ }_{\text {normal }}, 13$ of 30 for S2 ${ }_{\text {TCMR }}$, and 26 of 30 for S3 ${ }_{\mathrm{ABMR}}$. All top 30 transcripts correlating with the $\mathrm{S} 4_{\mathrm{UV}}$ scores and most of the top transcripts correlating with PC3 
Table 1. Demographics

\begin{tabular}{|c|c|}
\hline Biopsy characteristics & All (889 biopsies) \\
\hline \multicolumn{2}{|l|}{ Days to biopsy after transplant (TxBx) } \\
\hline Mean & 787 \\
\hline Median (range) & $232(6-10,150)$ \\
\hline \multicolumn{2}{|l|}{ Days to most recent follow-up after biopsy } \\
\hline Mean & 756 \\
\hline Median (range) & $385(1-3,854)$ \\
\hline \multicolumn{2}{|l|}{ Indication for biopsy } \\
\hline Clinical (\% of known) & $154(18 \%)$ \\
\hline Protocol biopsy (\% of known) & $613(70 \%)$ \\
\hline Follow-up (\% of known) & $108(12 \%)$ \\
\hline Not available (\% of total) & $14(2 \%)$ \\
\hline Patient characteristics & All (454 patients) \\
\hline Mean patient age at first biopsy (range) & $51(2-78)$ \\
\hline Mean donor age (range) & $41(6-68)$ \\
\hline \multicolumn{2}{|l|}{ Patient sex } \\
\hline Male (\% of known) & $321(71 \%)$ \\
\hline Female (\% of known) & $133(29 \%)$ \\
\hline \multicolumn{2}{|l|}{ Donor sex } \\
\hline Male (\% of known) & $286(66 \%)$ \\
\hline Female (\% of known) & $145(34 \%)$ \\
\hline Not available (\% of total) & $23(5 \%)$ \\
\hline Patient had a previous failed heart transplant & $13(3 \%)$ \\
\hline \multicolumn{2}{|l|}{ Heart status at last follow-up } \\
\hline Alive at last follow-up (\% of known) & $394(86 \%)$ \\
\hline Deceased (\% of known) & $56(13 \%)$ \\
\hline Failed and re-transplanted (\% of known) & $3(1 \%)$ \\
\hline Not available (\% of total) & $1(<1 \%)$ \\
\hline \multicolumn{2}{|l|}{ Primary disease $^{A}$} \\
\hline Dilated Cardiomyopathy (\% of known) & $229(50 \%)$ \\
\hline Hypertrophic Cardiomyopathy (\% of known) & $28(6 \%)$ \\
\hline Restrictive Cardiomyopathy (\% of known) & $11(2 \%)$ \\
\hline Other Cardiomyopathies & $37(8 \%)$ \\
\hline Congenital Heart Defect (\% of known) & $29(6 \%)$ \\
\hline Coronary Artery Disease (\% of known) & $85(19 \%)$ \\
\hline Other (\% of known) & $34(8 \%)$ \\
\hline Not available (\% of total) & $2(<1 \%)$ \\
\hline
\end{tabular}

were strongly expressed in macrophages, and many had been previously annotated as quantitative constitutive macrophage transcripts (QCMATs) (24) or alternatively activated macrophage transcripts (AMATs) (25). Six of the top $30 \mathrm{~S}_{\mathrm{UV}}$-correlated transcripts and 12 of the top 30 PC3-correlated transcripts had been annotated as inducible in injury systems: IRRATs in human kidney transplants $(21,26)$, IRIT5 in mouse kidney isografts (19), and cIRITs in mouse heart isografts (18).

The top 30 transcripts correlated with $\mathrm{PC} 1, \mathrm{PC} 2, \mathrm{~S}_{\text {normal }}, \mathrm{S} 2_{\mathrm{TCMR}}$, and $\mathrm{S} 3_{\mathrm{ABMR}}$ differed from those associated with $\mathrm{S}_{\mathrm{UV}}$ and PC3 (Supplemental Tables 3-7) and their cellular expression is summarized in Table 4. For $\mathrm{S} 1_{\text {normal }}$ and PC1, these included IFN- $\gamma$-inducible transcripts; for $\mathrm{S} 2_{\mathrm{TCMR}}$, effector $\mathrm{T}$ cell transcripts; and for $\mathrm{S} 3_{\mathrm{ABMR}}$ and PC2, endothelial transcripts, IFN- $\gamma$-inducible transcripts, and NK cell transcripts (similar to the published analysis of ABMR transcripts) (27). The top transcripts correlating with the $S 1_{\text {normal }}$ ' $\mathrm{S} 2_{\mathrm{TCMR}}$, and $\mathrm{S} 3_{\mathrm{ABMR}}$ scores in the 4AA model were similar to those published for the 3AA model (17).

We performed gene ontology (GO) biological process analysis for the top 100 unique transcripts most correlated with each archetype score (Table 5). S1 ${ }_{\text {normal }}$-associated transcripts reflected IFN- $\gamma$ effects, $\mathrm{S}_{\mathrm{TCMR}}$-associated transcripts reflected $\mathrm{T}$ cell activation, and $\mathrm{S} 3_{\mathrm{ABMR}}$ associated with angiogenesis and IFN- $\gamma$ effects. S4 $4_{\mathrm{UV}}$ associated with inflammation pathways, many expressed in neutrophils or granulocytes. The GO biological 
Table 2. Histology summary available in 889 EMBs

\begin{tabular}{|c|c|c|}
\hline \multicolumn{2}{|c|}{ Histology diagnoses ${ }^{A}$ ( $\%$ of known diagnoses) } & All (889 biopsies) \\
\hline \multicolumn{2}{|l|}{ No Rejection } & $334(38 \%)$ \\
\hline \multirow{2}{*}{ TCMR Related } & TCMR & $84(9 \%)$ \\
\hline & pTCMR & $273(31 \%)$ \\
\hline \multirow{2}{*}{ ABMR Related } & ABMR & $51(6 \%)$ \\
\hline & pABMR & $63(7 \%)$ \\
\hline \multirow{2}{*}{ Other } & ABMR/TCMR (Mixed) & $9(1 \%)$ \\
\hline & pABMR/pTCMR & $71(8 \%)$ \\
\hline \multicolumn{2}{|l|}{ Missing } & $4(0 \%)$ \\
\hline \multicolumn{2}{|c|}{ DSA Status } & All (454 patients) \\
\hline \multicolumn{3}{|c|}{ Last known DSA status at most recent biopsy } \\
\hline \multicolumn{2}{|l|}{ Positive } & $158(37 \%)$ \\
\hline \multicolumn{2}{|l|}{ Negative } & $267(63 \%)$ \\
\hline \multicolumn{2}{|l|}{ Not tested } & $29(6 \%)$ \\
\hline
\end{tabular}

ABiopsies in the 889 cohort were labeled as follows:
PAMR
.. No ABMR
pAMR1, pAMR1l+, pAMR1H+ ..... Possible ABMR (pABMR)
pAMR2, pAMR3
.... BMR
TCMROR
No TCMR
TCMR1R
Possible TCMR (pTCMR)
TCMR2R, TCMR3R
TCMR

Biopsies in the 331 cohort were reclassified using the above criteria.

${ }^{B}$ The most recent DSA status at time of most recent biopsy was used, if known. DSA statuses dated more than 14 days after the biopsy were not considered. If the most recent DSA status at time of biopsy was not known, but the patient was most recently PRA negative, the DSA status was presumed negative. PRA statuses dated more than 14 days after the biopsy were not considered.

processes associated with PC1-, PC2-, and PC3-associated transcripts (Supplemental Table 8) were similar to those associated with $\mathrm{S}_{\text {normal }}, \mathrm{S}_{\mathrm{ABMR}}$, and $\mathrm{S} 4_{\mathrm{UV}}$, respectively; $\mathrm{PC} 1$ associated with $\mathrm{T}$ cell-mediated inflammation and IFN- $\gamma$ effects, PC2 with angiogenesis, and PC3 with granulocyte activation pathways.

Expression of pathogenesis-based transcript sets. The published pathogenesis-based transcript (PBT) sets were empirically derived in primary human cell lines, mouse and human transplants, or through literature review to represent biological processes in rejection, inflammation, and/or injury (see Methods). We examined PBT scores in EMBs grouped by their highest archetype scores in normal, TCMR, ABMR, or UV groups (Table 6). PBT scores were expressed relative to the group of normal biopsies (those whose highest score was $\mathrm{S} 1_{\text {normal }}$ ).

As expected, TCMR biopsies had the highest scores for quantitative cytotoxic $\mathrm{T}$ cell transcripts (QCATs) and T cell burden transcripts (TCBs). ABMR biopsies had the highest scores for endothelial and non-endothelial DSA-selective transcripts (eDSASTs and DSASTs), and for NK cell burden transcripts (NKBs). TCMR had the highest IFN- $\gamma$-inducible transcripts (GRITs), but biopsies with high S4 $4_{\mathrm{UV}}$ scores also had high GRIT scores. All PBT scores related to injury (cIRITs, IRIT5s, IRRATs, and DAMPs) and macrophages (QCMATs and AMATs) were highest in biopsies whose highest score was $\mathrm{S} 4_{\mathrm{UV}}$, although TCMR biopsies also had high scores.

Relationships of injury and rejection transcripts to time of biopsy after transplant. Figure 3A examines the 100-biopsy moving averages of injury measurements ( $\mathrm{S}_{\mathrm{UV}}, \mathrm{PC} 3$, and 5 injury-related PBT sets) in relation to time of biopsy after transplant. The injury-related PBT scores were high in early biopsies — presumably reflecting the stress of donation and implantation - and diminished over time.

Figure $3 \mathrm{~B}$ shows the moving average of rejection scores $\mathrm{S} 2_{\mathrm{TCMR}}$ and $\mathrm{S} 3_{\mathrm{ABMR}}$, and includes the injury scores $\mathrm{S} 4_{\mathrm{UV}}$ and PC3 for ease of comparison. $\mathrm{S} 2_{\mathrm{TCMR}}$ was very low initially then showed 2 peaks before eventually declining: one at about 3 months after transplant and one after 2 to 3 years. S $3_{\mathrm{ABMR}}$ was also low initially and rose at about 3 years, then continued high. This temporal profile was similar to that of rejection reported for kidney transplants, where the late TCMR peak and the rise in ABMR correlate with nonadherence (28). 

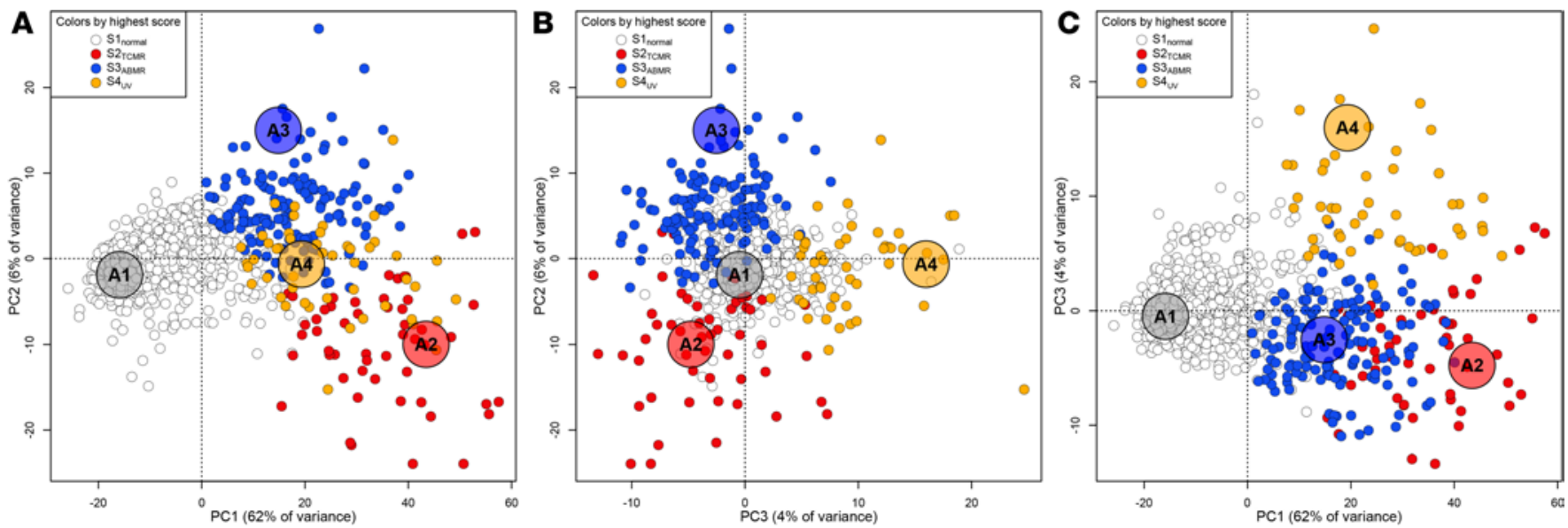

Figure 2. Principal component analysis of $\mathbf{8 8 9}$ heart transplant biopsies based on their expression of rejection-associated transcripts (RATs). Samples in $\mathbf{A}-\mathbf{C}$ are colored according to their highest archetype score (white $=\mathrm{S} 1_{\text {normal, }}$, red $=\mathrm{S} 2_{\text {TсMR }}$, blue $=\mathrm{S}_{{ }_{\mathrm{ABMR}}}$, orange $=\mathrm{S} 4_{\mathrm{UV}}$ ) in the 4 -archetype model trained on RAT expression. The large ghosted points labeled A1 (normal), A2 (TCMR), A3 (ABMR), and A4 (unexplained variance, UV) mark the positions of the theoretical archetypes to which each sample is compared.

The relationships between $\mathrm{S} 4_{\text {injury }}$ PC3, and time of biopsy after transplant are compatible with the temporal profile of resolution of injury due to donation-implantation stresses (28), and are very different from the rejection-related scores. Note that the TCMR peaks in Figure 3B corresponded with increases in some injury-related transcript sets in Figure 3A, e.g., DAMP, QCMAT, and IRRAT scores. This is expected given the ability of TCMR, an interstitial process, to produce parenchymal injury (see Table 5 above). In contrast, ABMR is an intracapillary process that usually spares the parenchyma until late stage. The results indicate that the UV described by PC3 and S4 relates to recent parenchymal injury, and we designated $\mathrm{S} 4_{\mathrm{UV}}$ as $\mathrm{S} 4_{\text {injury }}$.

Relating the molecular phenotype to LVEF. Figure 4 shows rolling average of left ventricular ejection fraction (LVEF) ( $y$ axis) versus increasing archetype scores ( $x$ axis). The scores from all 606 biopsies with LVEF scores were used in the calculations for each line, e.g., the $S 1_{\text {normal }}$ line uses all $606 \mathrm{~S}_{\text {normal }}$ scores. The rolling mean LVEF increased with increasing $\mathrm{S} 1_{\text {normal }}$ score $\left(P=8 \times 10^{-5}\right.$ by robust regression on the raw data $)$ and decreased with increasing $\mathrm{S} 2_{\mathrm{TCMR}}$ score $\left(P=1 \times 10^{-7}\right)$ and $\mathrm{S} 4_{\text {injury }}$ score $(P=0.002)$. S $3_{\mathrm{ABMR}}$ had no relationship with $\operatorname{LVEF}(P=0.94)$.

The loss of function could reflect parenchymal changes associated with injury. To explore this, we examined myosin and tropomyosin transcripts highly expressed (signal $>10,000$ ) in the normal EMBs (Table 7). All myosin and tropomyosin transcripts were decreased in biopsies with TCMR or injury, and to a lesser extent in ABMR biopsies. Expression of myosin and tropomyosin correlated with $\mathrm{S} 1_{\text {normal }}$ and anticorrelated with $\mathrm{S} 2_{\mathrm{TCMR}}$ and $\mathrm{S} 4_{\text {injury }}$. There was minimal correlation with $\mathrm{S} 3_{\mathrm{ABMR}}$.

Injury increases the probability that histology will diagnose rejection in biopsies with no molecular rejection. Among biopsies with low molecular rejection scores (i.e., unlikely to have rejection), those that had elevated $\mathrm{S} 4_{\text {injury }}$ scores $\left(\mathrm{S} 4_{\text {injury }} \geq 0.2\right)$ were more likely to be called rejection by histology than those with low $\mathrm{S} 4_{\text {injury }}$ scores (Table $8 ; P=1.2 \times 10^{-9}$, odds ratio 4.0 ). Thirty-three percent of the biopsies with high probability of molecular injury and low probability of molecular rejection were potentially misdiagnosed as severe rejection (ISHLT grades $2-3$ ) by histology, and 45\% were potentially misdiagnosed as mild rejection (ISHLT grade 1). Thus, in biopsies with a low probability of rejection by molecular assessments, rejection is diagnosed more frequently by histology when those biopsies have injury.

\section{Discussion}

Having previously developed a first-generation system for measuring rejection in EMBs, we undertook an unsupervised analysis of UV with the goal of understanding the molecular phenotype of cardiac parenchymal injury and distinguishing it from rejection. We also aimed to define the time course of rejection and injury phenotypes in the prevalent heart transplant population, and establish the relationship between molecular changes and function. Single EMB bites from heart transplant recipients in international centers were analyzed using Affymetrix microarrays in IRB-approved protocols. We explored unexplained 
Table 3. The top 30 transcripts correlating with the $\mathbf{5 4}$ uv score

\begin{tabular}{|c|c|c|c|}
\hline Gene Symbol & Gene Name & Spearman correlation with $\mathbf{5 4}$ & Principal expression in cell panel ${ }^{A}$ \\
\hline FCGR2A /R2C & $\begin{array}{l}\text { Fc fragment of IgG, low affinity Ila/llc, } \\
\text { receptor (CD32a/CD32c) }\end{array}$ & 0.76 & MP \\
\hline C3AR1 & Complement component 3a receptor 1 & 0.75 & MP \\
\hline CD14 & CD14 molecule & 0.74 & MP \\
\hline $\mathrm{C} 1 \mathrm{QB}^{\mathrm{B}}$ & Complement component 1, q subcomponent, B chain & 0.73 & MP, DC \\
\hline LILRA6/LILRB3 & $\begin{array}{c}\text { Leukocyte immunoglobulin-like receptor, subfamily A, } \\
\text { member } 6 \text { // subfamily B, member } 3\end{array}$ & 0.72 & MP \\
\hline TYROBP & TYRO protein tyrosine kinase binding protein & 0.72 & MP, DC, NK \\
\hline FCER1G & $\begin{array}{c}\text { Fc fragment of IgE, high affinity I, receptor for; } \gamma \\
\text { polypeptide }\end{array}$ & 0.72 & MP, DC, NK \\
\hline TLR2 & Toll-like receptor 2 & 0.71 & MP \\
\hline MSR1 & Macrophage scavenger receptor 1 & 0.71 & MP, DC \\
\hline C1QC & Complement component 1, q subcomponent, $\mathrm{C}$ chain & 0.71 & MP, DC \\
\hline FCGR1A ${ }^{B}$ & Fc fragment of IgG, high affinity la, receptor (CD64) & 0.71 & MP \\
\hline RNASE6 & Ribonuclease, RNase A family, k6 & 0.71 & MP, DC \\
\hline FCGR3A/R3B ${ }^{B}$ & $\begin{array}{l}\text { Fc fragment of IgG, low affinity IIIa/IIIb, } \\
\text { receptor (CD16a/CD16b) }\end{array}$ & 0.71 & MP, NK \\
\hline $\mathrm{C} 1 \mathrm{Q} \mathrm{A}^{\mathrm{B}}$ & Complement component 1, q subcomponent, A chain & 0.71 & MP, DC \\
\hline RBM47 & RNA binding motif protein 47 & 0.70 & MP, DC \\
\hline CYBB $^{B}$ & Cytochrome b-245, $\beta$ polypeptide & 0.70 & MP \\
\hline TBXAS1 & Thromboxane A synthase 1 (platelet) & 0.70 & MP \\
\hline MS4A4A & Membrane-spanning 4-domains, subfamily $\mathrm{A}$, member 4A & 0.70 & MP \\
\hline
\end{tabular}

${ }^{A}$ The cell panel was described previously (see Methods). Note the granulocytes were not represented in this cell panel, and expression in such cells was not assessed. ${ }^{B}$ Gene belonging to rejection-associated transcripts (RATs). MP, macrophage; DC, dendritic cell; NK, natural killer cell; B, B cell.

variance as PC3 and a new S4 ${ }_{\text {injury }}$ score in a 4AA model, which better explained the variance in abnormal EMBs than the $\mathrm{S} 2_{\text {TCMR }}$ and $\mathrm{S} 3_{\mathrm{ABMR}}$ scores did in the previous $3 \mathrm{AA}$ model (17). Both PC3 and $\mathrm{S} 4_{\text {injury }}$ scores correlated with macrophage transcripts, with injury-and-repair transcripts derived in injured human and mouse transplants with no rejection, and with DAMPs that represent cellular damage. The transcripts reflecting recent parenchymal injury were most disturbed immediately after transplant and regressed toward normal over several months, compatible with the temporal profile of donation-implantation injury resolution. Rejection, particularly TCMR, also induced injury molecules. Both high $\mathrm{S} 4_{\text {injury }}$ scores and high $\mathrm{S} 2_{\text {TCMR }}$ scores were associated with lower LVEF, whereas high $\mathrm{S} 1_{\text {normal }}$ scores were associated with higher LVEF. Failure to recognize injury appeared to lead to overestimates of rejection; biopsies with molecular injury but no molecular rejection were more often called rejection by histology. Thus, molecular diagnosis of rejection is improved when transcript expression reflecting injury is recognized, and the correlation of injury measurements with cardiac dysfunction makes EMBs more relevant to clinical scenarios.

Gene expression microarrays are the platform of choice for this stage of the project because they offer rapid translation from discovery to application. This project cannot be done with protein measurements because proteins cannot be amplified, and the products of many important transcripts would not be detectable by current measurement systems. At present, mRNA sequencing methods are unsuitable 
Table 4. Summary of annotation of cellular expression of the top 30 transcripts associated with scores in 889 EMBs

\begin{tabular}{|c|c|c|}
\hline Score $^{A}$ & Annotation of transcripts (number) based on highest cellular expression ${ }^{B}$ & Interpretation \\
\hline $\mathrm{S} 1_{\text {normal }}$ & 26 IFN- $\gamma$-inducible, 4 mononuclear cells (T, MMDC) & Absence of IFN- $\gamma$ effects, inflammation \\
\hline $\mathrm{S} 2_{\mathrm{TCMR}}$ & 28 effector T cells & TCMR \\
\hline $53_{\mathrm{ABMR}}$ & 10 endothelial (HUVEC), 14 IFN- $\gamma$-inducible, 4 NK & Endothelial changes, ABMR \\
\hline $54_{U V}$ & 30 macrophages & Injury-induced \\
\hline PC1 & 19 IFN- $\gamma$-inducible, 11 mononuclear cells (T, MMDC) & IFN- $\gamma$ effects, inflammation \\
\hline PC2 & 28 endothelial (HUVEC), 1 IFN- $\gamma$-inducible, 1 indeterminate & Endothelial changes \\
\hline
\end{tabular}

${ }^{A}$ Details in Tables 3 and 4, and in Supplemental Tables 2-6. ${ }^{\mathrm{B}}$, effector T cells; MMDC, monocytes, macrophages, or dendritic cells; NK, natural killer cells; HUVEC, human umbilical vein endothelial cell; IFN- $\gamma$, interferon $\gamma$.

for both discovery and application systems because the technology is too time, cost, and data intensive to provide the level of insight that microarrays do over large cohorts. However, RNA sequencing offers many potential insights at the discovery level that we are currently exploring, and may be an important diagnostic platform in the future. When the molecular algorithms are fully validated, we hope that the microarray system will be available through one or more central laboratories with rapid processing (shipping time plus $24-48$ hours), and in this way will be able to impact care and alter clinical practice, as is already proceeding for kidney biopsies (29).

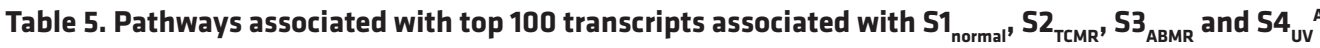

\begin{tabular}{|c|c|c|c|c|c|c|c|}
\hline \multicolumn{2}{|c|}{ Pathways associated with $\mathbf{S 1}_{\text {normal }}$} & \multicolumn{2}{|c|}{ Pathways associated with $\mathbf{S 2}_{\mathrm{TCMR}}$} & \multicolumn{2}{|c|}{ Pathways associated with $\mathrm{S}_{\text {ABMR }}$} & \multicolumn{2}{|c|}{ Pathways associated with $\mathbf{5 4}$ uv } \\
\hline Description & $\begin{array}{l}\text { Adjusted } P \\
\text { value }\end{array}$ & Description & $\begin{array}{l}\text { Adjusted } P \\
\text { value }\end{array}$ & Description & $\begin{array}{l}\text { Adjusted } P \\
\text { value }\end{array}$ & Description & $\begin{array}{l}\text { Adjusted } P \\
\text { value }\end{array}$ \\
\hline response to IFN- $\gamma$ & $7 \times 10^{-28}$ & T cell activation & $2 \times 10^{-45}$ & response to IFN- $\gamma$ & $3 \times 10^{-17}$ & neutrophil activation & $1 \times 10^{-24}$ \\
\hline $\begin{array}{l}\text { cellular response to } \\
\text { IFN- } \gamma\end{array}$ & $2 \times 10^{-26}$ & $\begin{array}{c}\text { regulation of } \\
\text { lymphocyte activation }\end{array}$ & $1 \times 10^{-29}$ & $\begin{array}{l}\text { IFN- } \gamma \text {-mediated } \\
\text { signaling pathway }\end{array}$ & $1 \times 10^{-12}$ & $\begin{array}{c}\text { neutrophil } \\
\text { degranulation }\end{array}$ & $4 \times 10^{-24}$ \\
\hline $\begin{array}{l}\text { leukocyte cell-cell } \\
\text { adhesion }\end{array}$ & $1 \times 10^{-16}$ & $\begin{array}{c}\text { regulation of } \\
\text { leukocyte activation }\end{array}$ & $2 \times 10^{-27}$ & $\begin{array}{l}\text { positive regulation of } \\
\text { response to external } \\
\text { stimulus }\end{array}$ & $2 \times 10^{-8}$ & $\begin{array}{l}\text { neutrophil-mediated } \\
\text { immunity }\end{array}$ & $7 \times 10^{-24}$ \\
\hline $\begin{array}{l}\text { antigen processing } \\
\text { and presentation of } \\
\text { exogenous peptide } \\
\text { antigen }\end{array}$ & $1 \times 10^{-15}$ & $\begin{array}{l}\text { leukocyte cell-cell } \\
\text { adhesion }\end{array}$ & $2 \times 10^{-26}$ & $\begin{array}{l}\text { type I interferon } \\
\text { signaling pathway }\end{array}$ & $2 \times 10^{-8}$ & $\begin{array}{l}\text { immune response- } \\
\text { activating signal } \\
\text { transduction }\end{array}$ & $4 \times 10^{-19}$ \\
\hline $\begin{array}{l}\text { antigen processing and } \\
\text { presentation of peptide } \\
\text { antigen }\end{array}$ & $3 \times 10^{-15}$ & $\begin{array}{l}\text { lymphocyte } \\
\text { differentiation }\end{array}$ & $7 \times 10^{-25}$ & $\begin{array}{l}\text { response to type I } \\
\text { interferon }\end{array}$ & $2 \times 10^{-8}$ & $\begin{array}{l}\text { regulation of innate } \\
\text { immune response }\end{array}$ & $5 \times 10^{-11}$ \\
\hline $\begin{array}{l}\text { antigen processing and } \\
\text { presentation }\end{array}$ & $3 \times 10^{-15}$ & $\begin{array}{l}\text { regulation of } \\
\text { leukocyte cell-cell } \\
\text { adhesion }\end{array}$ & $1 \times 10^{-23}$ & response to virus & $4 \times 10^{-8}$ & $\begin{array}{l}\text { adaptive immune } \\
\text { response }\end{array}$ & $5 \times 10^{-11}$ \\
\hline $\begin{array}{l}\text { regulation of } \\
\text { lymphocyte activation }\end{array}$ & $1 \times 10^{-14}$ & $\begin{array}{c}\text { regulation of cell-cell } \\
\text { adhesion }\end{array}$ & $6 \times 10^{-22}$ & cell chemotaxis & $4 \times 10^{-8}$ & $\begin{array}{l}\text { immune response- } \\
\text { regulating cell surface } \\
\text { receptor signaling } \\
\text { pathway }\end{array}$ & $1 \times 10^{-10}$ \\
\hline
\end{tabular}

${ }^{A}$ The 10 most significant gene ontology biological processes (BP) associated with each of the first 3 PCs of a RAT-based heart transplant biopsy principal component analysis (based on the top 100 unique transcripts). 
Table 6. Relative expression ${ }^{A}$ (fold increase vs. normal) in biopsies assigned to groups named on the basis of their highest archetype score

\begin{tabular}{|c|c|c|c|c|c|}
\hline & & $\begin{array}{c}\text { Normal } n=645 \\
\left(S 1_{\text {normal }}\right)\end{array}$ & $\begin{array}{c}\text { TCMR } n=52 \\
\left(S 2_{\text {TCMR }}\right)\end{array}$ & $\begin{array}{c}\text { ABMR } n=144 \\
\left(53_{A B M R}\right)\end{array}$ & $\begin{array}{c}\text { Unexplained } \\
\text { Variation } n=48 \\
\left(54_{\mathrm{uv}}\right)\end{array}$ \\
\hline \multicolumn{2}{|r|}{ Mean days after transplant (TxBx) } & 756 & 857 & 1073 & 216 \\
\hline \multirow{2}{*}{ TCMR-related } & Effector T cells (QCAT) & 1.00 & 3.88 & 2.13 & 2.40 \\
\hline & Effector $T$ cells ( $T$ cell burden) (TCB) & 1.00 & 5.61 & 2.22 & 2.43 \\
\hline ABMR-related & NK cells (NKB) & 1.00 & 1.42 & 1.76 & 1.28 \\
\hline $\begin{array}{l}\text { Rejection and/ } \\
\text { or injury }\end{array}$ & IFN- $\gamma$ inducible (GRIT) & 1.00 & 2.57 & 1.92 & 2.41 \\
\hline \multirow{3}{*}{ Injury-related } & Alternatively activated macrophage associated (AMAT) & 1.00 & 2.27 & 1.54 & 2.69 \\
\hline & Macrophage associated (OCMAT) & 1.00 & 1.96 & 1.39 & 2.40 \\
\hline & Injury-induced in mouse heart transplants (cIRIT) & 1.00 & 1.36 & 1.16 & 1.44 \\
\hline
\end{tabular}

AThe fold changes were calculated using biopsies with group $1(n=645)\left(S 1_{\text {normal }}\right)$ as a control. ${ }^{B}$ Algorithms and gene lists are available at https://www. ualberta.ca/medicine/institutes-centres-groups/atagc/research/gene-lists.

A number of observations argue that the UV detected by PC3 and S4 ${ }_{\text {injury }}$ reflects parenchymal injury and response to injury. PC3 and S4 ${ }_{\text {injury }}$ are highest in biopsies immediately after transplantation when rejection is uncommon on contemporary immunosuppression. Both PC3 and S4 ${ }_{\text {injury }}$ correlate with transcripts previously annotated in injured tissues, particularly macrophage transcripts and transcripts correlating with depressed function in injured kidney transplants and heart isografts with no rejection (IRRATs, IRIT5s, and cIRITs). PC3 and S4 $4_{\text {injury }}$ are associated with decreased LVEF. The possibility that $\mathrm{PC} 3$ and $\mathrm{S} 4_{\text {injury }}$ reflect rejection is not supported by the distinct time courses and transcript associations of these scores compared with the rejection scores. Nevertheless, some EMBs with rejection can also have high injury-related molecular scores, which is compatible with the concept that rejection can induce injury, and some EMBs display severe rejection and severe injury. Some high S4 ${ }_{\text {injury }}$ scores were in biopsies with rejection, particularly TCMR, which is an interstitial process that directly affects the parenchyma.

PC3 and S4 ${ }_{\text {injury }}$ were derived using unsupervised analysis of variation in RATs because RATs are increased by injury (albeit less than in rejection), due to the link between innate and adaptive immunity (16, 30), but that does not imply that RATs will be the transcripts most correlated with injury. In unsupervised analysis any set of molecules can be used to group biopsies, but the biologic processes in the biopsy groups is best understood by the actual transcripts most highly correlated with the groups, be they the molecules used to group the biopsies or others. In this study, the transcripts most highly expressed in biopsies with high S4 $4_{\text {injury }}$ and PC3 scores were actually those annotated as macrophage and injury transcripts, not RATs.

By distinguishing between rejection and injury, the present study offers insight into the distribution of phenotypes over time after transplant, and reveals similarities between the heart and kidney transplant populations. Injury in kidney and heart transplants is extensive immediately after transplant and regresses toward normal (26), and can reappear in severe rejection states, particularly TCMR. In kidney transplants, TCMR and ABMR differ in their time course, with TCMR peaking earlier, but both emerge between 1 and 5 years after the first year at a time of high risk of nonadherence (28). TCMR becomes infrequent after $5-10$ years $(28,31-33)$, whereas ABMR is the dominant late-rejection phenotype. The relatively low incidence of late TCMR suggests adaptations in the effector $\mathrm{T}$ cell response, perhaps related to immunologic checkpoints (32).

Heart-kidney similarities do not obscure major differences, particularly in the parenchymal consequences of injury and rejection. We believe that cardiac parenchyma is inherently more fragile than kidney, and evokes a more inflammatory response. For example, TCMR in heart transplants is associated 


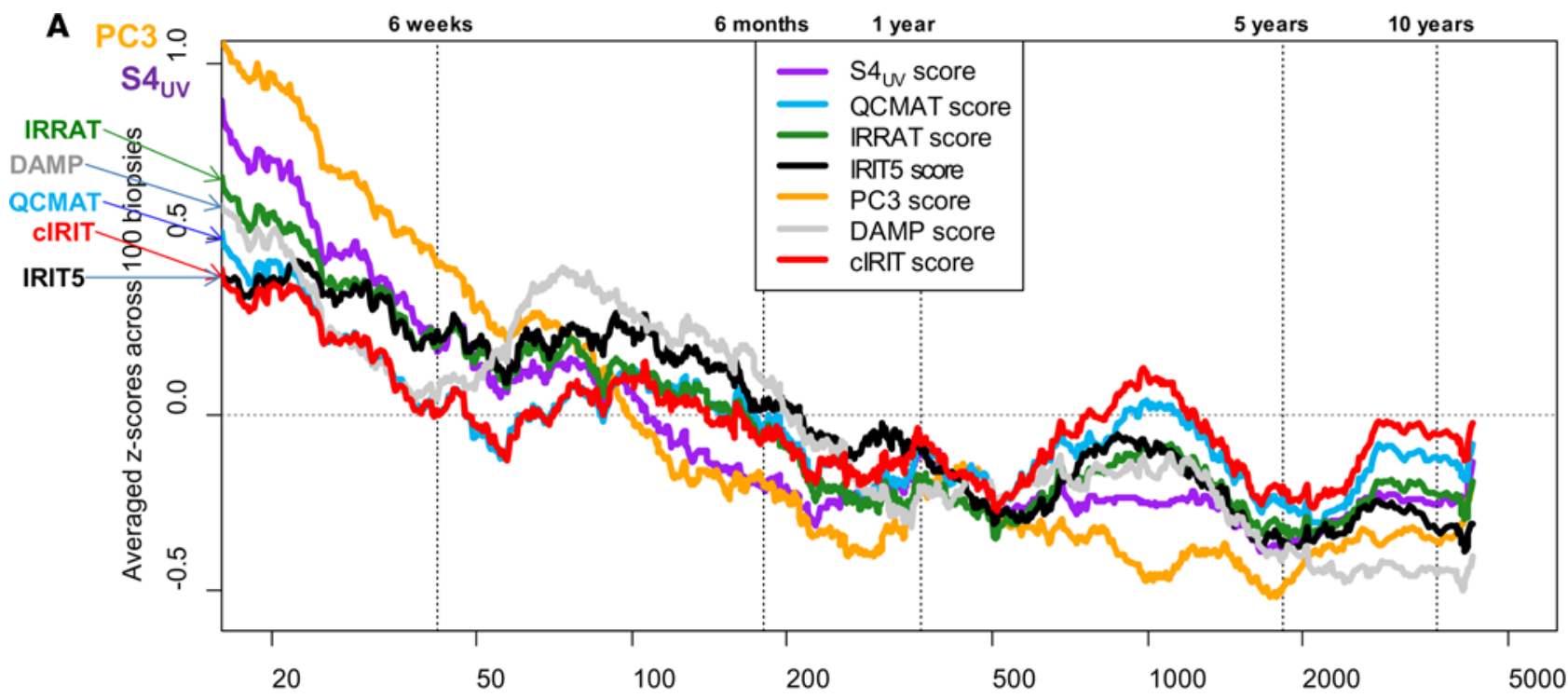

Time post-transplant to biopsy (days)

B

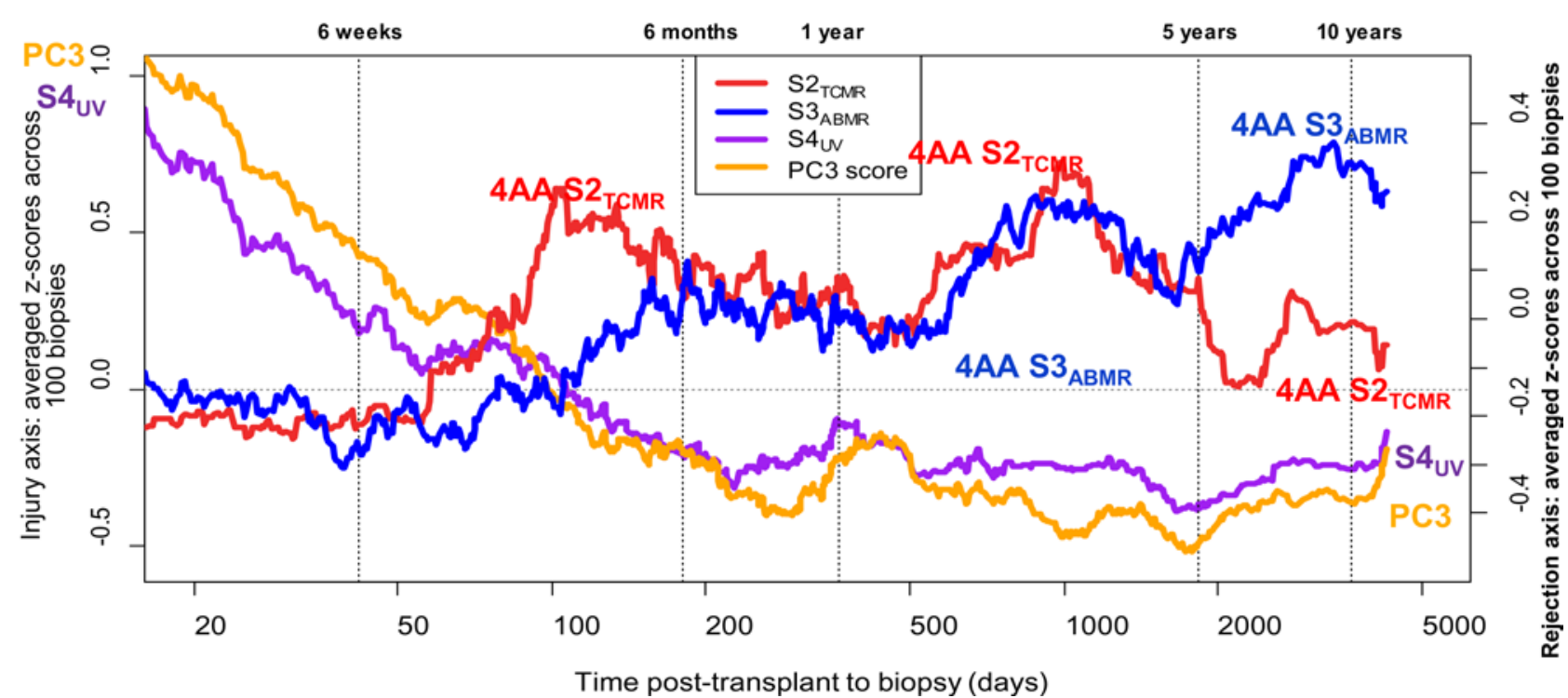

Figure 3. Moving average of standardized pathogenesis-based transcript (PBT) scores and archetype scores in biopsies ordered by increasing time after transplant (period $\mathbf{=} \mathbf{1 0 0}$ biopsies). The $\mathrm{S}_{\text {injury }}$ score is compared to injury PBT scores $(\mathbf{A})$ and rejection-related scores (B). Time after transplant is given in days, reported on a logarithmic scale. The $\mathrm{S2}_{\mathrm{TCMR}}, \mathrm{S3}_{\mathrm{ABMR}}$, and $\mathrm{S}_{\mathrm{UV}}$ scores are all taken from the 4-archetype model (4AA). PC3, principal component 3 from principal component analysis of 889 EMBs based on rejection-associated transcript expression; IRIT5, 5-day injury-and-repair-induced kidney transcripts; cIRIT, cardiac injury-and-repair-induced transcripts; IRRATs, kidney injury-and-repair-associated transcripts; DAMP, damage-associated molecular pattern transcripts; QCMATs, macrophage-associated transcripts; QCATs, effector T cell-associated transcripts.

with parenchymal necrosis (1), which is not a prominent feature of renal transplant TCMR (34). We have seen differences when comparing mouse heart and kidney transplant models; mouse kidney allografts (35) tolerate TCMR better than heart allografts in the same combination (unpublished studies). Thus, while similarities between responses to injury or rejection in different organs permit development of molecular assessment systems through unsupervised analysis, understanding how each organ responds uniquely will refine molecular assessment for those organs.

The extensive injury changes in EMB and the association of these changes with reduced function adds a new dimension to our understanding of the clinical scenarios in heart transplants. The response to wounding is shared across tissues and species, as illustrated by the DAMP transcripts, which are modulated by injury even in plants (36). Indeed, the response to wounding is a feature of cancer, "the wound that does not heal" $(37,38)$. 


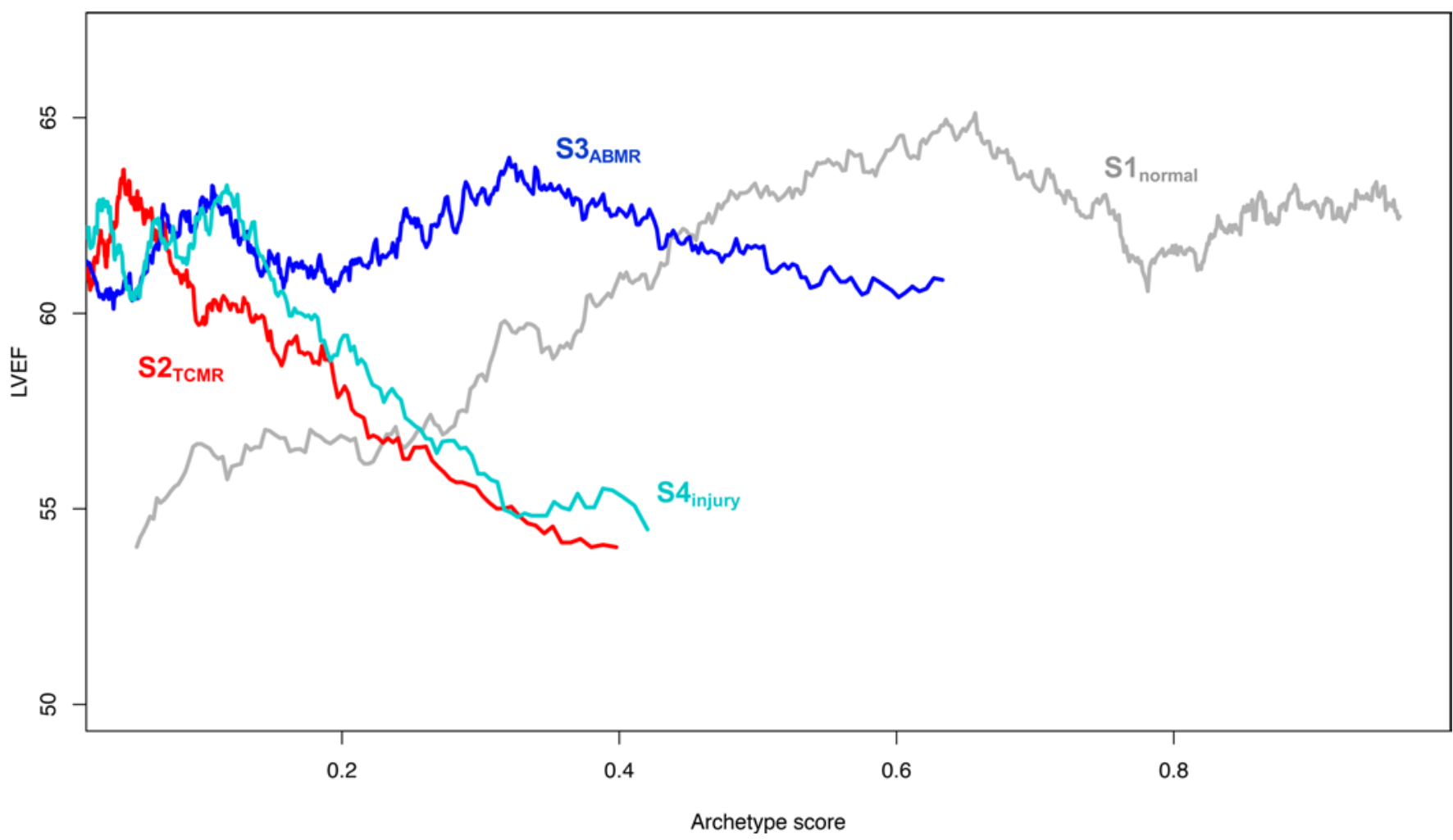

Figure 4. Running average of LVEF versus archetype scores. For each of the 4 archetype scores, the 606 biopsies with available LVEF data were sorted by the archetype score being plotted. A sliding window of size $n=85$ biopsies was then used to plot the mean LVEF versus mean archetype score. For example, the first data point on the left on the $\mathrm{S}_{\text {normal }}$ line corresponds to the mean LVEF and mean $\mathrm{S} 1_{\text {normal }}$ of the first through 85 th biopsies (sorted in ascending order of the $606 \mathrm{S1}_{\text {normal }}$ scores), the second point to the second through 86th biopsies, etc. The lines have different $x$-axis ranges because, for example, the highest $85 \mathrm{~S}_{\mathrm{TCMR}}$ scores are approximately 0.4 , while the highest 85 scores for each of $\mathrm{S1}_{\text {normal, }}, \mathrm{S3}_{\mathrm{ABMR}}$, and $\mathrm{S} 4_{\text {injury }}$ are larger.

Macrophage infiltration occurs within 3 days in injured organ isografts due to donation-implantation stress in the absence of allograft rejection $(19,20)$, accompanied by parenchymal changes as a component of healing $(16,30)$. Brain death has complex effects on cardiac hemodynamics and expression of inflammatory mediators $(39,40)$, and has the potential to increase the immunogenicity of the heart and increase the probability of rejection. As we move forward, it will be interesting to follow outcomes, including cardiac allograft vasculopathy, in biopsies with different combinations of injury and rejection.

Study limitations include lack of diastolic function parameters that may highlight additional clinical phenotypes, because rejection is believed to start with diastolic dysfunction (41). We did not consider clinical presentation or symptoms as phenotypes because such details are difficult to record and accurately quantify in an international multicenter study. Another dimension that can be examined is the relationship between ischemic times and $\mathrm{S} 4_{\text {injury }}$ scores in early postoperative biopsies. The $\mathrm{S} 4_{\text {injury }}$ score in early biopsies can be useful in comparing alternative preservation strategies. In later biopsies the source of injury is often probably rejection, particularly TCMR, but it is likely that there are other late injury phenotypes that have been missed and called rejection. Moreover, rejection-induced injury may persist after treatment of rejection and mistakenly be called "refractory rejection," causing unnecessary treatment. It is conceivable that extensive injury obscures molecular rejection, and we are currently developing binary classifiers to address this distinction in such biopsies (unpublished observations).

The availability of a platform that can measure parenchymal injury opens opportunities to explore the inflammatory and injury phenotypes in primary heart diseases that diffusely affect the parenchyma, such as myocarditis, with the expectation that injury features will also probably correlate with impaired LVEF in such

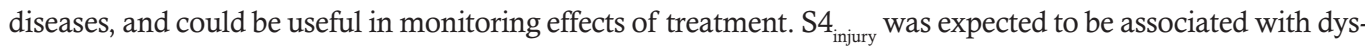
function because it correlates with IRRATs, which were originally defined by their association with poor function in recent kidney transplants $(21,26)$. The lower LVEF in biopsies with TCMR or injury may be related to myocyte response to injury, since myosin and tropomyosin transcripts were lower in biopsies with TCMR and 
Table 7. Relationship of archetype scores to myosin and tropomyosin mRNA expression ${ }^{A}$

\begin{tabular}{|c|c|c|c|c|c|c|c|c|c|c|c|c|c|}
\hline \multirow{2}{*}{$\begin{array}{l}\text { Affymetrix } \\
\text { designation }\end{array}$} & \multirow{2}{*}{$\begin{array}{c}\text { Gene } \\
\text { Symbol }\end{array}$} & \multirow[b]{2}{*}{ Gene Name } & \multicolumn{4}{|c|}{$\begin{array}{l}\text { Biopsies grouped by } \\
\text { highest archetype score }\end{array}$} & \multicolumn{7}{|c|}{$\begin{array}{c}\text { Correlation (Spearman) of gene expression with archetype } \\
\text { and PCA scores in } 889 \text { biopsies }\end{array}$} \\
\hline & & & $\begin{array}{c}\text { Normal } \\
n=645 \\
\left(51_{\text {normal }}\right)\end{array}$ & $\begin{array}{c}\text { TCMR } \\
n=52 \\
\left(52_{\text {TCMR }}\right)\end{array}$ & $\begin{array}{c}\text { ABMR } \\
n=144 \\
\left(S 3_{\text {ABMR }}\right)\end{array}$ & $\begin{array}{c}\text { Injury } \\
n=48 \\
\left(54_{\text {Injury }}\right)\end{array}$ & $\mathbf{S} 1_{\text {normal }}$ & $\mathbf{S 2} \mathbf{T C M R}$ & $53_{\text {ABMR }}$ & $54_{\text {injury }}$ & PC1 & PC2 & PC3 \\
\hline 11718277_a_at & MYL2 & myosin light chain 2 & 16,962 & 13,567 & 15,000 & 11,641 & 0.27 & -0.22 & 0.01 & -0.16 & -0.29 & 0.11 & -0.04 \\
\hline 11719790_a_at & MYL3 & myosin light chain 3 & 15,911 & 12,745 & 13,849 & 11,445 & 0.39 & -0.27 & -0.07 & -0.29 & -0.41 & 0.06 & -0.11 \\
\hline AVERACE & & & $\underline{16,437}$ & $\underline{13,156}$ & $\underline{14,424}$ & $\underline{11,543}$ & $\underline{0.33}$ & $\underline{-0.24}$ & $\underline{-0.03}$ & $\underline{-0.23}$ & $\underline{-0.35}$ & $\underline{0.09}$ & $\underline{-0.07}$ \\
\hline 11740313_s_at & $\begin{array}{l}\text { MYH6, } \\
\text { MYH7 }\end{array}$ & myosin, heavy chain $6, \alpha$ & & & & & & & & & & & \\
\hline $\begin{array}{l}\text { myosin, heavy } \\
\text { chain } 7, \beta\end{array}$ & 14,539 & 10,888 & 12,596 & 9,839 & 0.34 & -0.36 & -0.05 & -0.19 & -0.37 & 0.16 & 0.05 & & \\
\hline 11717570_s_at & $\mathrm{MYH7}$ & myosin, heavy chain $7, \beta$ & 17,992 & 14,847 & 16,137 & 13,201 & 0.34 & -0.35 & 0.00 & -0.25 & -0.38 & 0.19 & -0.03 \\
\hline AVERAGE & & & $\underline{16,266}$ & $\underline{12,867}$ & $\underline{14,367}$ & $\underline{11,520}$ & $\underline{0.34}$ & $\underline{-0.36}$ & $\underline{-0.02}$ & $\underline{-0.22}$ & $\underline{-0.37}$ & $\underline{0.17}$ & $\underline{0.01}$ \\
\hline 11738892_a_at & TPM1 & tropomyosin $1(\alpha)$ & 20,895 & 18,462 & 19,108 & 16,668 & 0.28 & -0.24 & -0.04 & -0.13 & -0.30 & 0.06 & 0.03 \\
\hline 11738893_s_at & TPM1 & tropomyosin $1(\alpha)$ & 15,270 & 12,644 & 13,700 & 11,575 & 0.37 & -0.23 & -0.06 & -0.22 & -0.38 & 0.04 & -0.01 \\
\hline 11742308_s_at & TPM1 & tropomyosin $1(\alpha)$ & 15,235 & 12,693 & 13,712 & 11,646 & 0.35 & -0.22 & -0.04 & -0.22 & -0.36 & 0.05 & -0.03 \\
\hline 11742309_x_at & TPM1 & tropomyosin $1(\alpha)$ & 12,396 & 10,191 & 11,191 & 9,381 & 0.35 & -0.31 & -0.08 & -0.15 & -0.37 & 0.09 & 0.12 \\
\hline AVERAGE & & & $\underline{15,949}$ & $\underline{13,498}$ & $\underline{14,428}$ & $\underline{12,318}$ & $\underline{0.34}$ & -0.25 & -0.06 & -0.18 & -0.35 & $\underline{0.06}$ & $\underline{0.03}$ \\
\hline
\end{tabular}

AProbesets for myosin light chains and heavy chains and for tropomyosin genes were selected only on the bases of high expression values $(>10,000)$ in normal biopsies.

injury, recalling the loss of the heart transcripts in inflamed hearts (18) and loss of transcripts associated with differentiated function in inflamed kidney transplants (19-21). Such loss of transcripts associated with highly differentiated parenchyma could reflect either dedifferentiation or dilution by transcripts from inflammatory cells, but our kidney studies indicate the major mechanism is likely due to dedifferentiation (42). Heart transplant EMBs represent a new window on important problems such as the role of macrophages in human cardiac injury, repair, remodeling, and heart failure - a dynamic and emerging topic in cardiology (43-48). The injury or death of cardiac myocytes attracts and activates macrophages, which then clear debris, promote healing, and potentially extend injury in some circumstances. The macrophage is a fundamental component of the cardiac response to wounding, which has been difficult to study in human hearts but can be explored in transplant EMBs in the MMDx system. Many organs probably share this response, and both features unique to the cardiac parenchyma and those shared by all tissues will be of interest. A key question for future studies is whether macrophages invading cardiac tissue in response to injury are merely associated with dysfunction, a contributor to dysfunction, or an important element in restoration of function and healing, and to define the mechanisms involved. The dualistic role of macrophages as both positive and negative agents involved in cardiac repair makes it difficult to draw actionable conclusions until these issues are resolved.

\section{Methods}

Population. The present observational study comparing MMDx analysis to standard-of-care assessments was approved by the ethics board of each center and is registered at Clinicaltrials.gov (NCT02670408). As previously described (17), biopsies were collected prospectively either for clinical indications or by

Table 8. Histologic rejection diagnoses in $\mathbf{5 8 6}$ biopsies with no molecular rejection, comparing absence versus presence of molecular injury

No molecular injury ${ }^{A}$ No molecular rejection No. $\quad$ Molecular injury ${ }^{A}$ No molecular rejection No. Biopsies ( $\%$ of column) $n=517$ biopsies ( $\%$ of column) $n=69$

$\begin{array}{lcc}\text { Histologic No Rejection } & 272(53 \%) & 15(22 \%) \\ \text { Histologic Possible Rejection } & 204(39 \%) & 31(45 \%) \\ \text { Histologic Rejection } & 41(8 \%) & 23(33 \%)\end{array}$

${ }^{\mathrm{A}}$ No molecular injury, no molecular rejection $=\mathrm{S} 4_{\text {injury }}<0.2, \mathrm{~S}_{\mathrm{TCMR}}<0.2, \mathrm{~S}_{\mathrm{ABMR}}<0.3$; molecular injury, no molecular rejection $=\mathrm{S} 4_{\text {injury }} \geq 0.2, \mathrm{~S} 2_{\mathrm{TCMR}}<0.2$, $\mathrm{S3}_{\text {ABMR }}<0.3$. Fisher's $P$ value $=1.2 \times 10^{-9}$, odds ratio 4.0 when histologic possible rejection and histologic rejection rows are combined. 
local protocol and processed for histology and HLA antibody testing as per local standard of care. Local histology assessment followed ISHLT guidelines $(1,6)$. The 889 biopsies from 455 patients included 18 taken as biological replicates from the same patient at the same time. Excluding these replicate biopsies did not influence the results. The 889 biopsies included 331 biopsies used in the previous study on molecular assessment of EMBs (17) plus 558 later biopsies.

Microarray analysis. As detailed elsewhere (17), purified total RNA from EMBs including RNA available from 97 Edmonton samples used in a previous study (18) was labeled with the 3' IVT Plus kit (Affymetrix) and hybridized to PrimeView microarrays (Affymetrix) according to manufacturer protocols (www. affymetrix.com).

Assignment of rejection diagnoses for comparison of molecular and histologic assessments. The centers often used different versions of the ISHLT classification as their histology standard of care. Therefore, to compare histologic and molecular rejection diagnoses, histology grades were translated into diagnoses of ABMR, possible ABMR (pABMR), TCMR, possible TCMR (pTCMR), combinations thereof, and no rejection. Histologic ABMR grades $1 \mathrm{H}+$ and $1 \mathrm{I}+$ were labeled pABMR, ABMR grades 2 and 3 were labeled ABMR, TCMR grade $1 R$ was labeled pTCMR, and grades $2 R$ and $3 R$ were labeled TCMR. Samples with a grade of 0 were labeled no rejection.

Selection of the RATs in kidney biopsies. The algorithms used to derive the RATs in kidney biopsies are published (16). RATs were the union of the top 200 probe sets associated by $P$ value with each of the 3 comparisons: all rejection versus everything else (rejection-RATs), ABMR versus everything else (ABMRRATs), or TCMR versus everything else (TCMR-RATs).

PBTs. The PBT sets include transcripts that reflect biological processes and cell types that play important roles in rejection. The PBTs were defined in human cell lines, mouse experimental models, and human transplant biopsies, and are described in detail on the ATAGC home page (https://www. ualberta.ca/medicine/institutes-centres-groups/atagc/research/gene-lists). For application to human heart biopsies, all PBT sets were filtered by interquartile range (IQR) for variance in 889 EMBs (IQR $>0.5$ ). PBT expression is summarized as a single score (geometric mean PBT expression in the sample divided by geometric mean PBT expression across samples in a control group, or standardized relative to the control group) based on their expression in any given biopsy. We examined PBT scores in relation to other phenotypes in the EMBs. We focused on PBTs for injury-and-repair transcripts expressed in acute kidney injury (IRRATs; derived in human kidney transplant biopsies) $(21,26)$, injury-repairinduced transcripts (IRIT5s; derived in day 5 mouse kidney isografts) (19), quantitative constitutive macrophage transcripts (QCMATs; derived in primary human cells) (24), alternatively activated macrophage transcripts (AMATs; derived in mouse models of ischemic necrosis) (25), DSA-selective transcripts (DSASTs; derived in human kidney transplant biopsies) (49), NK cell burden transcripts (NKBs; derived in primary human cells) (49), T cell burden transcripts (TCBs; derived in primary human cells) (49), quantitative cytotoxic T cell transcripts (QCATs; derived in primary human cells) (50), IFN $\gamma-$ inducible transcripts (GRITs; derived in primary human cells) (51), cardiac injury-and-repair-induced transcripts (cIRITs; derived in day 5 mouse heart isografts) (18), and damage-associated molecular pattern transcripts (DAMPs; derived from literature) $(18,22,36)$.

Expression in cell panel. The principal expression of transcripts was annotated from a primary human cell panel previously described (52), as well as by previous annotation in PBT lists.

\section{Statistics}

$P C A$. PCA is a method of dimensionality reduction that derives a set of linearly uncorrelated variables (PC scores), which explain most of the variance in a data set. We performed unsupervised PCA of the EMBs based on RAT expression. We focused on PC1, PC2, and PC3 scores, which reflect the top 3 aspects of variation in the data.

Archetypal analysis. The archetypes package in $\mathrm{R}$ was used (53). Archetypal analysis (54) is a clustering-like method of unsupervised analysis that extrapolates a predefined $k$ number of theoretical biopsies (archetypes, denoted by "A") that represent major idealized phenotypes in the data set. Each biopsy is scored in terms of how closely it relates to each archetype. The $k$ archetype scores (denoted by "S") assigned to each biopsy describes its weighted distance from each of the $k$ theoretical archetypes such that the sum of scores is 1 . In this investigation, we studied heart biopsies in terms of 3-archetype (3AA) and 4-archetype (4AA) models trained on RAT expression. Both models assign scores representing no rejection $\left(\mathrm{S} 1_{\text {normal }}\right)$, 
$\operatorname{TCMR}\left(\mathrm{S} 2_{\mathrm{TCMR}}\right)$, and $\mathrm{ABMR}\left(\mathrm{S} 3_{\mathrm{ABMR}}\right)$. The $4 \mathrm{AA}$ model identifies an additional dimension of unexplained variance $\left(\mathrm{S} 4_{\mathrm{UV}}\right)$, which we characterized in this paper. Biopsies could be assigned to groups according to their highest archetype score.

Relationship between LVEF and the archetype scores. Six hundred and six biopsies had LVEF data at the time of biopsy. For each of the 4 archetype scores from the 4AA model, the biopsies were first sorted in ascending order using that archetype's scores. These were then used to calculate running averages (window size $=85$ biopsies) of LVEF versus archetype score based on the 606 sorted scores for each archetype in turn. Robust regression was performed using the $1 \mathrm{mRob}$ function in the $\mathrm{R}$ package robust, using the raw data (not the running averages).

Study approvals. The study protocol was approved by the University of Alberta Research Ethics Board (Pro00022227), as well as by the research ethics boards of all of the contributing centers. All biopsies were collected with written informed consent.

\section{Author contributions}

PFH was the principal investigator for this study. JR and MDP analyzed data and prepared figures and tables. JR, AZA, MC, MGCL, MD, ECD, JG, XJ, DHK, JK, AL, PM, LP, and AZ were involved with biopsy collection and manuscript revision. $\mathrm{PFH}$ and $\mathrm{MDP}$ wrote the manuscript.

\section{Acknowledgments}

The authors thank Jessica Chang for preparing and editing the final version of the manuscript. This research has been supported by funding and/or resources from Genome Canada, Canada Foundation for Innovation, the University Of Alberta Hospital Foundation, the Alberta Ministry of Advanced Education and Technology, the Mendez National Institute of Transplantation Foundation, and the Roche Organ Transplant Research Foundation. PF Halloran held a Canada Research Chair in Transplant Immunology until 2008 and currently holds the Muttart Chair in Clinical Immunology. Parts of this study were presented at the 2018 ISHLT meeting in Nice, France on April 11-12, 2018.

Address correspondence to: Philip F. Halloran, Alberta Transplant Applied Genomics Centre, 250 Heritage Medical Research Centre, University of Alberta, Edmonton, AB T6G 2S2, Canada. Phone: 780.492.6160; Email: phallora@ualberta.ca.

1. Stewart S, et al. Revision of the 1990 working formulation for the standardization of nomenclature in the diagnosis of heart rejection. J Heart Lung Transplant. 2005;24(11):1710-1720.

2. Billingham M, Kobashigawa JA. The revised ISHLT heart biopsy grading scale. J Heart Lung Transplant. 2005;24(11):1709.

3. Mehra MR, et al. The 2016 International Society for Heart Lung Transplantation listing criteria for heart transplantation: A 10-year update. J Heart Lung Transplant. 2016;35(1):1-23.

4. Leone O, et al. 2011 consensus statement on endomyocardial biopsy from the Association for European Cardiovascular Pathology and the Society for Cardiovascular Pathology. Cardiovasc Pathol. 2012;21(4):245-274.

5. Berry GJ, et al. The ISHLT working formulation for pathologic diagnosis of antibody-mediated rejection in heart transplantation: evolution and current status (2005-2011). J Heart Lung Transplant. 2011;30(6):601-611.

6. Berry GJ, et al. The 2013 International Society for Heart and Lung Transplantation Working Formulation for the standardization of nomenclature in the pathologic diagnosis of antibody-mediated rejection in heart transplantation. J Heart Lung Transplant. 2013;32(12):1147-1162.

7. Bruneval P, et al. The XIIIth Banff Conference on Allograft Pathology: The Banff 2015 Heart Meeting Report: Improving antibody-mediated rejection diagnostics: strengths, unmet needs, and future directions. Am J Transplant. 2017;17(1):42-53.

8. Fedrigo M, et al. Inflammatory cell burden and phenotype in endomyocardial biopsies with antibody-mediated rejection (AMR): a multicenter pilot study from the AECVP. Am J Transplant. 2015;15(2):526-534.

9. Tible M, et al. Pathologic classification of antibody-mediated rejection correlates with donor-specific antibodies and endothelial cell activation. J Heart Lung Transplant. 2013;32(8):769-776.

10. Loupy A, et al. Complement-binding anti-HLA antibodies and kidney-allograft survival. N Engl J Med. 2013;369(13):1215-1226

11. Gebel HM, Bray RA. In search of perfection. Am J Transplant. 2013;13(8):1951-1952.

12. Visentin J, et al. Denatured class I human leukocyte antigen antibodies in sensitized kidney recipients: prevalence, relevance, and impact on organ allocation. Transplantation. 2014;98(7):738-744.

13. Gombos $\mathrm{P}$, et al. Influence of test technique on sensitization status of patients on the kidney transplant waiting list. Am J Transplant. 2013;13(8):2075-2082.

14. Reeve J, et al. Molecular diagnosis of T cell-mediated rejection in human kidney transplant biopsies. Am J Transplant. 2013;13(3):645-655.

15. Crespo-Leiro MG, et al. Concordance among pathologists in the second Cardiac Allograft Rejection Gene Expression Observational Study (CARGO II). Transplantation. 2012;94(11):1172-1177. 
16. Halloran PF, Venner JM, Famulski KS. Comprehensive analysis of transcript changes associated with allograft rejection: combining universal and selective features. Am J Transplant. 2017;17(7):1754-1769.

17. Halloran PF, et al. Building a tissue-based molecular diagnostic system in heart transplant rejection: The heart Molecular Microscope Diagnostic (MMDx) System. J Heart Lung Transplant. 2017;36(11):1192-1200.

18. Mengel M, et al. The molecular phenotype of heart transplant biopsies: relationship to histopathological and clinical variables. Am J Transplant. 2010;10(9):2105-2115.

19. Famulski KS, et al. Transcriptome analysis reveals heterogeneity in the injury response of kidney transplants. Am J Transplant. 2007;7(11):2483-2495.

20. Einecke G, Mengel M, Hidalgo L, Allanach K, Famulski KS, Halloran PF. The early course of kidney allograft rejection: defining the time when rejection begins. Am J Transplant. 2009;9(3):483-493.

21. Famulski KS, et al. Molecular phenotypes of acute kidney injury in kidney transplants. J Am Soc Nephrol. 2012;23(5):948-958

22. Land WG, Agostinis P, Gasser S, Garg AD, Linkermann A. Transplantation and damage-associated molecular patterns (DAMPs). Am J Transplant. 2016;16(12):3338-3361.

23. Heil M, Land WG. Danger signals - damaged-self recognition across the tree of life. Front Plant Sci. 2014;5:578.

24. Famulski KS, Sis B, Billesberger L, Halloran PF. Interferon-gamma and donor MHC class I control alternative macrophage activation and activin expression in rejecting kidney allografts: a shift in the Th1-Th2 paradigm. Am J Transplant. 2008;8(3):547-556.

25. Famulski KS, et al. Defining the canonical form of T-cell-mediated rejection in human kidney transplants. Am J Transplant. 2010;10(4):810-820.

26. Venner JM, Famulski KS, Reeve J, Chang J, Halloran PF. Relationships among injury, fibrosis, and time in human kidney transplants. JCI Insight. 2016;1(1):e85323.

27. Loupy A, et al. Gene expression profiling for the identification and classification of antibody-mediated heart rejection. Circulation. 2017;135(10):917-935.

28. Sellarés J, et al. Understanding the causes of kidney transplant failure: the dominant role of antibody-mediated rejection and nonadherence. Am J Transplant. 2012;12(2):388-399.

29. Halloran PF, et al. Real time central assessment of kidney transplant indication biopsies by microarrays: The INTERCOMEX Study. Am J Transplant. 2017;17(11):2851-2862.

30. Halloran PF, et al. Review: The transcripts associated with organ allograft rejection. Am J Transplant. 2018;18(4):785-795.

31. Halloran PF, Reeve JP, Pereira AB, Hidalgo LG, Famulski KS. Antibody-mediated rejection, T cell-mediated rejection, and the injury-repair response: new insights from the Genome Canada studies of kidney transplant biopsies. Kidney Int. 2014;85(2):258-264

32. Halloran PF, et al. Disappearance of T cell-mediated rejection despite continued antibody-mediated rejection in late kidney transplant recipients. J Am Soc Nephrol. 2015;26(7):1711-1720.

33. Einecke G, Reeve J, Halloran PF. A molecular biopsy test based on arteriolar under-hyalinosis reflects increased probability of rejection related to under-immunosuppression. Am J Transplant. 2018;18(4):821-831.

34. Haas M, et al. The Banff 2017 Kidney Meeting Report: Revised diagnostic criteria for chronic active T cell-mediated rejection, antibody-mediated rejection, and prospects for integrative endpoints for next-generation clinical trials. Am J Transplant. 2018;18(2):293-307.

35. Jabs WJ, et al. Heterogeneity in the evolution and mechanisms of the lesions of kidney allograft rejection in mice. Am J Transplant. 2003;3(12):1501-1509.

36. Heil M, Land WG, Tör M. Editorial: Wound recognition across the tree of life. Front Plant Sci. 2016;7:1319.

37. Feng Y, Santoriello C, Mione M, Hurlstone A, Martin P. Live imaging of innate immune cell sensing of transformed cells in zebrafish larvae: parallels between tumor initiation and wound inflammation. PLoS Biol. 2010;8(12):e1000562.

38. Riss J, et al. Cancers as wounds that do not heal: differences and similarities between renal regeneration/repair and renal cell carcinoma. Cancer Res. 2006;66(14):7216-7224.

39. Wilhelm MJ, Pratschke J, Laskowski IA, Paz DM, Tilney NL. Brain death and its impact on the donor heart-lessons from animal models. J Heart Lung Transplant. 2000;19(5):414-418.

40. Wilhelm MJ, et al. Activation of the heart by donor brain death accelerates acute rejection after transplantation. Circulation. 2000;102(19):2426-2433.

41. Badano LP, et al. European Association of Cardiovascular Imaging/Cardiovascular Imaging Department of the Brazilian Society of Cardiology recommendations for the use of cardiac imaging to assess and follow patients after heart transplantation. Eur Heart J Cardiovasc Imaging. 2015;16(9):919-948.

42. Einecke G, Broderick G, Sis B, Halloran PF. Early loss of renal transcripts in kidney allografts: relationship to the development of histologic lesions and alloimmune effector mechanisms. Am J Transplant. 2007;7(5):1121-1130.

43. Schirone L, et al. A review of the molecular mechanisms underlying the development and progression of cardiac remodeling. Oxid Med Cell Longev. 2017;2017:3920195.

44. Tourki B, Halade G. Leukocyte diversity in resolving and nonresolving mechanisms of cardiac remodeling. FASEB J. 2017;31(10):4226-4239.

45. Tham YK, Bernardo BC, Ooi JY, Weeks KL, McMullen JR. Pathophysiology of cardiac hypertrophy and heart failure: signaling pathways and novel therapeutic targets. Arch Toxicol. 2015;89(9):1401-1438.

46. Montecucco F, Liberale L, Bonaventura A, Vecchiè A, Dallegri F, Carbone F. The role of inflammation in cardiovascular outcome. Curr Atheroscler Rep. 2017;19(3):11.

47. Frangogiannis NG. Inflammation in cardiac injury, repair and regeneration. Curr Opin Cardiol. 2015;30(3):240-245.

48. Glezeva N, Horgan S, Baugh JA. Monocyte and macrophage subsets along the continuum to heart failure: Misguided heroes or targetable villains? J Mol Cell Cardiol. 2015;89(Pt B):136-145.

49. Hidalgo LG, Sellares J, Sis B, Mengel M, Chang J, Halloran PF. Interpreting NK cell transcripts versus T cell transcripts in renal transplant biopsies. Am J Transplant. 2012;12(5):1180-1191.

50. Hidalgo LG, et al. The transcriptome of human cytotoxic T cells: measuring the burden of CTL-associated transcripts in human kidney transplants. Am J Transplant. 2008;8(3):637-646.

51. Famulski KS, et al. Changes in the transcriptome in allograft rejection: IFN-gamma-induced transcripts in mouse kidney 
allografts. Am J Transplant. 2006;6(6):1342-1354.

52. Venner JM, Famulski KS, Badr D, Hidalgo LG, Chang J, Halloran PF. Molecular landscape of T cell-mediated rejection in human kidney transplants: prominence of CTLA4 and PD ligands. Am J Transplant. 2014;14(11):2565-2576.

53. Eugster MJA, Leish F. From Spider-Man to Hero - archetypal analysis in R. J Stat Softw. 2009;30(8):1-23.

54. Cutler A, Breiman L. Archetypal analysis. Technometrics. 1994;36:338-347. 